\title{
Clinical Characteristics of Children with Coronavirus Disease 2019 in Hubei, China
}

Fang $Z_{H E N G}{ }^{1 \dagger}$, Chun LIAO $^{2 \dagger}$, Qi-hong FAN $^{3 \dagger}$, Hong-bo CHEN ${ }^{1 \dagger}$, Xue-gong ZHAO ${ }^{2}$, Zhong-guo XIE ${ }^{3}, \mathrm{Xi}^{-l i n} \mathrm{LI}^{4}$, $\mathrm{Chun}^{-x i}$ $\mathrm{CHEN}^{4}$, Xiao-xia LU ${ }^{5}$, Zhi-sheng LIU ${ }^{5}$, Wei LU ${ }^{6}$, Chun-bao CHEN ${ }^{6}$, Rong JIAO ${ }^{7}$, Ai-ming ZHANG ${ }^{7}$, Jin-tang WANG ${ }^{8}, \mathrm{Xi}^{-}$-wei DING $^{8}$, Yao-guang ZENG ${ }^{9}$, Li-ping CHENG ${ }^{9}$, Qing-feng HUANG ${ }^{10}$, Jiang WU ${ }^{11}$, Xi-chang LUO ${ }^{11}$, Zhu-jun WANG ${ }^{1}$, Yan-yan $\mathrm{ZHONG}^{12}$, Yan BAI ${ }^{1 \#}$, Xiao-yan WU ${ }^{1 \#}$, Run-ming JIN ${ }^{1 \#}$

${ }^{1}$ Department of Pediatrics, Union Hospital, Tongji Medical College, Huazhong University of Science and Technology, Wuhan 430022, China

${ }^{2}$ Department of Pediatrics, Yichang No. 3 People's Hospital, Yichang 443000, China

${ }^{3}$ Department of Pediatrics, Jingzhou First People's Hospital, Jingzhou 434000, China

${ }^{4} X i s h u i$ People's Hospital, Huanggang 438000, China

${ }^{5}$ Wuhan Children's Hospital, Tongji Medical College, Huazhong University of Science and Technology, Wuhan 430015, China

${ }^{6}$ Department of Pediatrics, Yichang Central Hospital, Yichang 443000, China

${ }^{7}$ Department of Pediatrics, Xiangyang First People's Hospital, Xiangyang 441000, China

${ }^{8}$ Department of Pediatrics, Shiyan People's Hospital, Shiyan 442000, China

${ }^{9}$ Department of Pediatrics, Huanggang Central Hospital, Huanggang 438000, China

${ }^{10}$ Department of Pediatrics, Jiangling People's Hospital, Jingzhou 434101, China

${ }^{11}$ Huangshi Maternity and Child Health Care Hospital, Huangshi 435000, China

${ }^{12}$ Department of Pediatrics, Huazhong University of Science and Technology Hospital, Wuhan 430074, China

(C) Huazhong University of Science and Technology 2020

\begin{abstract}
Summary: Since December 2019, COVID-19 has occurred unexpectedly and emerged as a health problem worldwide. Despite the rapidly increasing number of cases in subsequent weeks, the clinical characteristics of pediatric cases are rarely described. A cross-sectional multicenter study was carried out in 10 hospitals across Hubei province. A total of 25 confirmed pediatric cases of COVID-19 were collected. The demographic data, epidemiological history, underlying diseases, clinical manifestations, laboratory and radiological data, treatments, and outcomes were analyzed. Of 25 hospitalized patients with COVID-19, the boy to girl ratio was 1.27:1. The median age was 3 years. COVID-19 cases in children aged $<3$ years, 3-6 years, and $\geq 6$-years patients were 10 $(40 \%), 6(24 \%)$, and $9(36 \%)$, respectively. The most common symptoms at onset of illness were fever (13 [52\%]), and dry cough (11 [44\%]). Chest CT images showed essential normal in 8 cases (33.3\%), unilateral involvement of lungs in 5 cases $(20.8 \%)$, and bilateral involvement in 11 cases (45.8\%). Clinical diagnoses included upper respiratory tract infection $(n=8)$, mild pneumonia $(n=15)$, and critical cases $(n=2)$. Two critical cases $(8 \%)$ were given invasive mechanical ventilation, corticosteroids, and immunoglobulin. The symptoms in $24(96 \%)$ of 25 patients were alleviated and one patient had been discharged. It was concluded that children were susceptible to COVID-19 like adults, while the clinical presentations and outcomes were more favorable in children. However, children less than 3 years old accounted for majority cases and critical cases lied in this age group, which demanded extra attentions during home caring and hospitalization treatment.
\end{abstract}

Key words: SARS-CoV-2; COVID-19; coronavirus; children

Since December 2019, an epidemic caused by severe acute respiratory syndrome coronavirus 2 (SARS-CoV-2) infection has occurred unexpectedly

Fang ZHENG, E-mail: fangzheng99@sina.cn; Chun LIAO, E-mail: 1c7spring@163.com; Qi-hong FAN, E-mail: 1187286305@qq.com; Hong-bo CHEN, E-mail: hbchen@hust.edu.cn ${ }^{\dagger}$ These authors contributed equally to the work. \#Corresponding authors, Yan BAI, E-mail: yanbaixh@hust. edu.cn; Xiao-yan WU, E-mail: xwu@hust.edu.cn; Run-ming JIN, E-mail: jinrunm@qq.com in Wuhan, Hubei Province, China, and it had quickly spread from Wuhan to the other areas in China and abroad $^{[1-4]}$. The World Health Organization named this novel coronavirus disease, COVID-19, which was known as the novel conoravirus pneumonia (NCP) in China. Full-genome sequencing analysis indicated that SARS-CoV-2 has $89 \%$ nucleotide identity with bat SARS-like-CoVZXC21 and $82 \%$ with that of human SARS-CoV ${ }^{[5]}$. According to the updated information from National Health Commission of the People's Republic China, by February 15, 2020, the current epidemic status has shown a total of 69110 confirmed cases in the worldwide, and 68584 confirmed cases, 
8228 suspected cases with 1666 deaths reported around 31 provinces and cities in $\mathrm{China}^{[6]}$. In recent weeks, emerging studies have reported that most patients with COVID-19 had an epidemiological history including a travel or residence history in Wuhan City and neighboring areas, and contacting with confirmed or suspected $\operatorname{cases}^{[7]}$. The main clinical features of adult patients included fever, dry cough, dyspnea, myalgia, fatigue, normal leukocyte counts or leukopenia, and radiographic evidence of pneumonia ${ }^{[8]}$. COVID-19 was more likely to affect older men with comorbidities and could result in acute respiratory distress syndrome (ARDS) ${ }^{[9]}$. Wang et $a l^{[10]}$ elaborated hospital-related transmission of COVID-19 was suspected in $41 \%$ of patients, $26 \%$ of patients received ICU care, and mortality was $4.3 \%$. Compared to adults, the number of reported pediatric patients is limited ${ }^{[11,12]}$, and the clinical characteristics of pediatric COVID-19 was rarely reported. The objective of this study is to describe the clinical characteristics of 25 hospitalized pediatric COVID-19.

\section{PARTICIPANTS AND METHODS}

\subsection{Study Design and Participants}

We did a retrospective study including data from an established network of 10 purposely selected public hospitals situated in Wuhan urban and peri-urban areas of Wuhan. This case series was approved by the institutional ethics board of Tongji Medical College, Huazhong University of Science and Technology. Inpatient records included all children aged 1 month-14 years admitted to hospital with COVID-19 between February 1, 2020, and February 10, 2020. Diagnostic criteria for COVID-19 were determined according to the recommendation for the diagnosis and treatment of novel coronavirus infection in children in Hubei (Trial version 1$)^{[13]}$. Oral consent was obtained from patients' parents.

\subsection{Data Collection}

The medical records of patients were analyzed by a trained team of physicians from the department of pediatrics in ten hospitals. All patients were confirmed by the test positive for SARS-CoV-2. Demographic data, medical history, epidemiological history, underlying diseases, clinical symptoms, signs, laboratory data, radiological characteristics, treatments and outcomes were obtained with collection forms from electronic medical records. Epidemiological history was confirmed if they met any one of the following criteria: (1) children with travel or residence history in Wuhan City and neighboring areas, or other areas with persistent local transmission within 14 days prior to disease onset; (2) children with a history of contact with patients with fever or respiratory symptoms who had travel or residence history in Wuhan City and neighboring areas, or in other areas with persistent local transmission within 14 days prior to disease onset; (3) children with a history of contact with confirmed or suspected cases of COVID-19 within 14 days prior to disease onset; and (4) children who were related with a cluster outbreak.

\subsection{Procedures}

Throat swab and/or nasal-pharyngeal swab samples were collected for extracting RNA from suspected patients with SARS-CoV-2 infection. After collection, the samples were placed into a collection tube with 150 $\mu \mathrm{L}$ of virus preservation solution, and total RNA was extracted within $2 \mathrm{~h}$ using the respiratory sample RNA isolation kit (Zhongzhi, Wuhan, China). The detailed procedures had been described by Huang et $a l^{[7]}$. These diagnostic criteria were determined according to the recommendation by the National Institute for Viral Disease Control and Prevention, China.

Lab investigations included a complete blood count and serum biochemical tests for liver function, lactate dehydrogenase, electrolytes, coagulation, and C-reactive protein (CRP). Nasal and pharyngeal throat swabs were tested for common pathogens, including influenza A and B virus, respiratory syncytial virus, adenovirus, parainfluenza virus, mycoplasma pneumoniae, and chlamydia pneumoniae using realtime RT-PCR assays approved by the China Food and Drug Administration. Routine bacterial examinations were also performed.

Patients were performed on chest computer tomography (CT) scans. Radiological diagnosis was reviewed by two experienced chest radiologists.

\subsection{Statistical Analysis}

Continuous variables were described using mean, median, and interquartile range (IQR) values. Categorical variables were described as percentages and compared using the $\chi^{2}$ test, although the Fisher exact test was used when data were limited. Continuous variables were compared using independent group $t$ tests when the data were normally distributed. Otherwise, the Mann-Whitney test was used. All statistical analyses were analyzed using Graph Pad Prism 6.0 software (USA). A two-sided P of less than 0.05 was considered statistically significant.

\section{RESULTS}

\subsection{Demographics Characteristics}

A total of 25 patients with COVID-19 were included in this study. There were $14(56 \%)$ males and $11(44 \%)$ females with the boy to girl ratio being 1.27:1. The median age was 3 years (IQR, $2-9$ years; range, 3 months -14 years). Children aged $<3$ years, $3-6$ years, and $\geq 6$ years were $10(40 \%), 6(24 \%)$, and 9 (36\%) respectively. Most patients were previously healthy and only 2 had underlying disease. One 
patient had congenital heart diseases, malnutrition, and suspected hereditary metabolic diseases. The other one had congenital heart disease. Both of them had already undergone operations.

\subsection{Epidemiological and Clinical Characteristics}

Twenty-one (84\%) had epidemiological contact history and only 4 patients didn't have any epidemiological history (table 1). Clinical diagnoses included upper respiratory tract infection (URI) $(n=8)$, mild pneumonia $(n=15)$, and critical cases $(n=2) .14$ of 25 patients $(56 \%)$ aged less than 6 years had mild illness. Patients had an uneven age distribution. The high incidence of infection was noted to be aged $<3$ years (40\%) (fig. 1). The most common symptoms at onset of illness were fever [13 (52\%)], followed by dry cough [11 (44\%)], diarrhea [3 (12\%)], nasal congestion [2 (8\%)], dyspnea [2 (8\%)], abdominal pain [2(8\%)], and vomiting [2 (8\%)] (table 2).

Table 1 Demographic and epidemiologic characteristics of 25 pediatric patients with COVID-19

\begin{tabular}{lc}
\hline Characteristics & Value \\
\hline Age (years) & $3(2-9)$ \\
$\quad$ Median (IQR) & \\
$\quad$ Subgroup, No. (\%) & $10(40)$ \\
1 month-3 years & $6(24)$ \\
$\quad 3-6$ years & $9(36)$ \\
$\quad \geq 6$ years & $14 / 11$ \\
Boy/girl & \\
Underling diseases, No. (\%) & $2(8)$ \\
$\quad$ Congenital heart disease & $23(92)$ \\
$\quad$ Previously healthy & \\
Epidemiological history & $5(16)$ \\
$\quad$ Travel or residence history in Wuhan City & \\
$\quad$ and neighboring areas & \\
$\quad$ Contact confirmed or suspected cases & $16(64)$ \\
$\quad$ infected with SARS-CoV-2 & $16(64)$ \\
$\quad$ Related with a familial cluster outbreak & $4(16)$ \\
$\quad$ Missing & $25(100)$ \\
\hline Hospitalization, No. (\%)
\end{tabular}

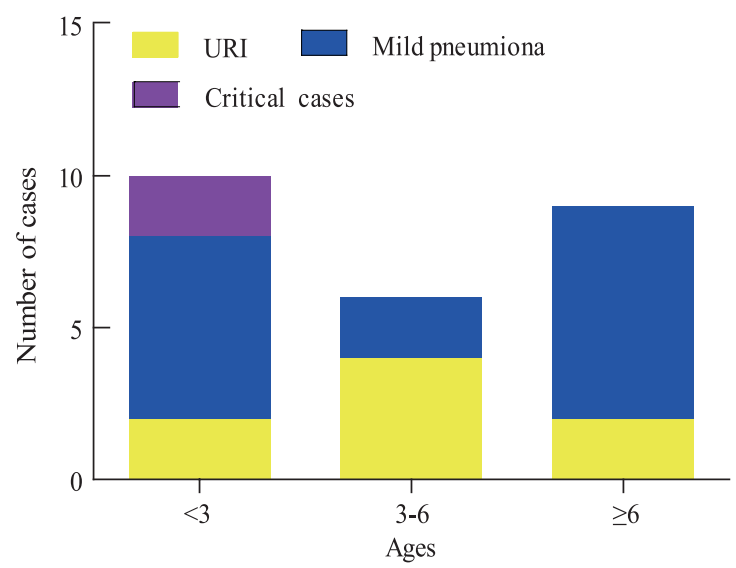

Fig. 1 Number of cases among different age groups with symptoms

\subsection{Laboratory Parameters and Imaging Features}

The blood counts of children on admission showed that the median level of white blood cell count was $6.2 \times 10^{9} / \mathrm{L}$ (IQR 4.30-9.85). The median level of lymphocyte count was $2.19 \times 10^{9} / \mathrm{L}(\mathrm{IQR}$ 1.15-3.31), and 10 of 25 patients showed lymphopenia (less than age-related reference values). The median values of CRP were $14.5 \mathrm{mg} / \mathrm{L}$ (IQR 0.93-25.04). Liver function test and myocardial enzyme assay had been done in 12 children. These tests revealed median alanine aminotransferase level of $12 \mathrm{U} / \mathrm{L}(10-13)$ and median creatine kinase isoenzyme level of $19 \mathrm{U} / \mathrm{L}$ (1336) (table 2). Except for the 2 critical cases, patients had normal renal function and coagulation function. Both 2 critical cases had high levels of serum lactic dehydrogenase. One critical patient was complicated with hyponatremia (table 3).

Table 2 Clinical characteristics and selected laboratory parameters of 25 pediatric patients with COVID-19

\begin{tabular}{ll}
\hline Characteristics & \multicolumn{1}{c}{ Value } \\
\hline Clinical symptom, No. (\%) & $13(52)$ \\
$\quad$ Fever & $2(8)$ \\
$\quad$ Nasal congestion & $11(44)$ \\
$\quad$ Cough & $2(8)$ \\
$\quad$ Dyspnea & $2(8)$ \\
$\quad$ Abdominal pain & $2(8)$ \\
$\quad$ Vomiting & $3(12)$ \\
$\quad$ Diarrhea & $8(32)$ \\
Clinical classifications, No. (\%) & $15(60)$ \\
$\quad$ Upper respiratory infection & $2(8)$ \\
$\quad$ Mild pneumonia & $6.2(4.30-9.85)$ \\
$\quad$ Critical cases & $2.19(1.15-3.31)$ \\
White blood cells (10/L) (Median, IQR) \\
Lymphocytes (10/L) (Median, IQR) \\
Alanine aminotransferase (U/L) & $12(10-13)$ \\
(Median, IQR) & $19(13-36)$ \\
Creatine kinase isoenzyme (U/L) & $14.5(0.93-25.04)$ \\
(Median, IQR) & \\
C-reaction protein (mg/L) (Median, IQR) & \\
Pathogens, No. (\%) & $25(100)$ \\
$\quad$ SARS-CoV-2 & $2(8)$ \\
Influenza virus type B & $3(12)$ \\
Mycoplasma pneumonia & $1(4)$ \\
Bacteria & $8(32)$ \\
Chest CT findings, No. (\%) & $5(20)$ \\
Normal images & $12(48)$ \\
Unilateral involvement & \\
Bilateral involvement & \\
\hline
\end{tabular}

"Normal reference values: white blood cells, (3.5-9.5) $\times 10^{9} / \mathrm{L}$; lymphocytes, $(2.1-5.7) \times 10^{9} / \mathrm{L}$ ( $<3$ years), $(1.4-4.2) \times 10^{9} / \mathrm{L}(4-6$ years), $(1.1-3.2) \times 10^{9} / \mathrm{L}$ ( $\geq 6$ years); alanine aminotransferase, 9-50 U/L; creatine kinase isoenzyme, $<25 \mathrm{U} / \mathrm{L}$; C-reactive protein, $<10 \mathrm{mg} / \mathrm{mL}$

All cases were confirmed as SARS-CoV-2 infection by virus nucleic acid test. Other identified pathogens included mycoplasma pneumoniae $(3 / 25,12 \%)$, influenza virus type B $(2 / 25,8 \%)$, and Enterobacter aerogenes $(1 / 25,8 \%)$. 
Table 3 Clinical characteristics and selected laboratory parameters of 2 critical cases of COVID-19

\begin{tabular}{|c|c|c|}
\hline Characteristics & Patient 1 & Patient 2 \\
\hline$\overline{\text { Age }}$ & 8 months & 1 year \\
\hline Boy/Girl & Boy & Boy \\
\hline Underling diseases & $\begin{array}{l}\text { Congenital heart diseases, malnutrition, } \\
\text { suspected hereditary metabolic diseases }\end{array}$ & Congenital heart diseases \\
\hline Epidemiological history & Travel in Wuhan city & Residence in Wuhan city \\
\hline Clinical symptoms & Cough, dyspnea, fever & Fever, diarrhea, dyspnea \\
\hline \multicolumn{3}{|l|}{ Laboratory examinations } \\
\hline White blood cells $\left(10^{9} / \mathrm{L}\right)$ & 4.43 & 11.96 \\
\hline Lymphocytes $\left(10^{9} / \mathrm{L}\right)$ & 1.46 & 2.47 \\
\hline Haemoglobin $(g / L)$ & 153 & 100 \\
\hline Platelet count $\left(10^{9} / \mathrm{L}\right)$ & 202 & 184 \\
\hline Alanine aminotransferase (U/L) & 12 & 20 \\
\hline Albumin $(g / L)$ & 30 & 30.1 \\
\hline Creatine kinase isoenzyme (U/L) & 98.8 & 62 \\
\hline Prothrombin time (s) & 14 & 14.3 \\
\hline Lactate dehydrogenase (U/L) & 609 & 361 \\
\hline Creatinin $(\mu \mathrm{mol} / \mathrm{L})$ & 45 & 224.5 \\
\hline Potassium (mmol/L) & 3.5 & 4.45 \\
\hline Sodium $(\mathrm{mmol} / \mathrm{L})$ & 136 & 126 \\
\hline C-reaction protein $(\mathrm{mg} / \mathrm{L})$ & 0.5 & 24.6 \\
\hline Pathogens & SARS-CoV-2 & SARS-CoV-2, Enterobacter aerogenes \\
\hline \multicolumn{3}{|l|}{ Treatment } \\
\hline Antiviral therapy & Interferon, oseltamivir & Interferon, oseltamivir \\
\hline Antibiotics application & Cefoperazone/sulbactam & Meropenem, linezolid \\
\hline Immunoglobulin & $2 \mathrm{~g} / \mathrm{kg}$ & $2 \mathrm{~g} / \mathrm{kg}$ \\
\hline Corticosteroid & $2 \mathrm{mg} / \mathrm{kg}$ & $2 \mathrm{mg} / \mathrm{kg}$ \\
\hline Respiratory support & Invasive mechanical ventilation & Invasive mechanical ventilation \\
\hline Blood purification & No & CVVHDF, PE \\
\hline Outcome & Partly alleviated & Significantly alleviated \\
\hline
\end{tabular}

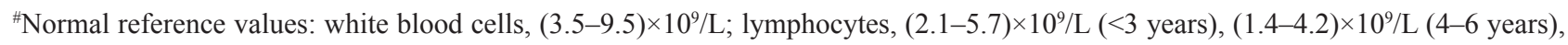
(1.1-3.2) $\times 10^{9} / \mathrm{L}$ ( $\geq 6$ years); haemoglobin, $130-175 \mathrm{~g} / \mathrm{L}$; platelet count, (125-350) $\times 10^{9} / \mathrm{L}$; alanine aminotransferase, 9-50 U/L; albumin, $35-55 \mathrm{~g} / \mathrm{L}$; creatine kinase isoenzyme, $<25 \mathrm{U} / \mathrm{L}$; prothrombin time, 9.4-12.5 s; lactate dehydrogenase, 125-243 U/L; creatinin, 44-133 $\mu \mathrm{mol} / \mathrm{L}$; potassium, 3.5-5.5 mmol/L; sodium, 136-145 mmol/L; C-reactive protein, $<10 \mathrm{mg} / \mathrm{mL}$.

CVVHDF, continuous veno-venous hemodiafiltration; PE, plasma exchange

On admission, 24 patients were subjected to chest CT scans. Chest CT images showed essential normal in 8 cases $(33.3 \%)$, unilateral involvement in 5 cases (20.8\%) and bilateral involvement in 11 cases $(45.8 \%)$ (table 2). The typical findings of chest CT images were bilateral patchy shadows or lung consolidations. An analysis of the age distribution of chest CT images showed that bilateral lung involvement was present in about $70 \%$ of children with aged $<3$ years, which was the highest percentage among the different age groups. The percentage of unilateral lesion and normal lungs were higher in children with aged $\geq 6$ years than other age groups (fig. 2). Radiographic images of the 2 critical cases on admission revealed bilateral lung consolidations (fig. 3).

\subsection{Treatment and Clinical Outcomes}

Patients were quarantined in the designated hospitals. 12 patients (48\%) received antiviral therapy (Interferon, arbidol, oseltamivir, lopinavir/litonavir). For antiviral therapy, interferon was the most widely

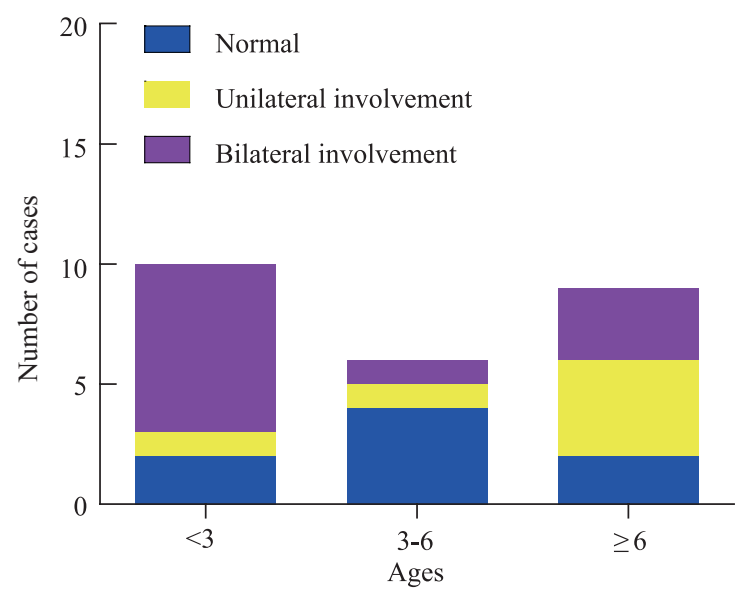

Fig. 2 An analysis of the age distribution by chest CT involvement

used in $12(48 \%)$ of patients. 13 patients (56\%) were treated with empirical antibiotics, and one patient showed bacteriological efficacy following treatment. 2 critical cases $(8 \%)$ were additionally given invasive 

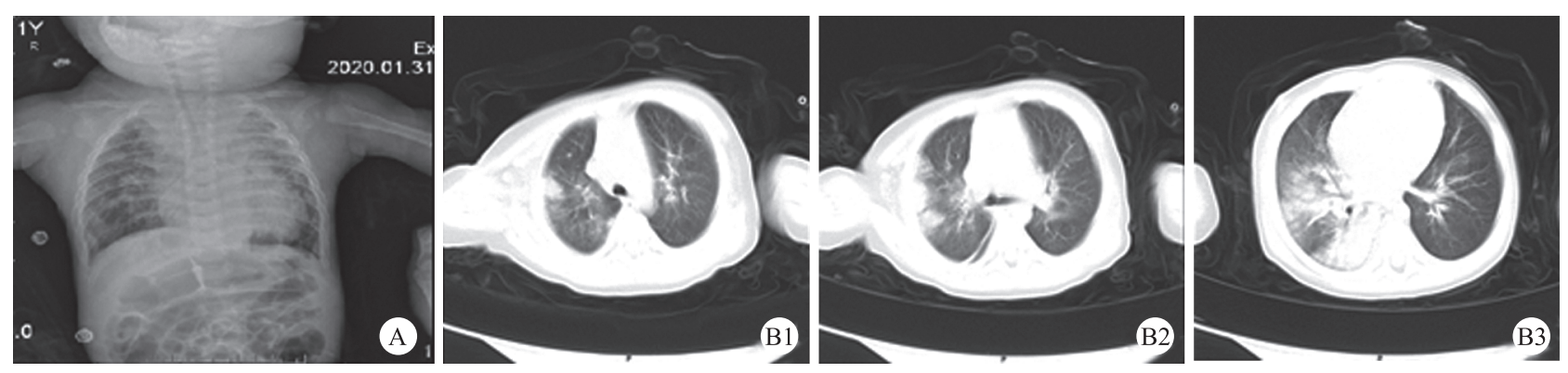

Fig. 3 Radiographic images of two critical cases on admission

A: Chest X-ray images from an 8-month-old boy showing bilateral multiple lobular and consolidation; B1-B3: Chest CT images of different layers from a 1-year-old boy showing bilateral ground glass opacity and subsegmental areas of consolidation

mechanical ventilation, systematic corticosteroids, and intravenous immunoglobulin. 1 of the 2 critical cases received kidney replacement therapy (table 3 ). As of February 15, 2020, one patient completely recovered and had been discharged. The symptoms on admission were alleviated in $24(96 \%)$.

\section{DISCUSSION}

Here, we report a cohort of 25 patients with confirmed COVID-19. Most children were previously healthy and had epidemiological contact history. The majority of pediatric patients had mild symptoms. Common symptoms at admission were fever and dry cough. However, a proportion of patients presented initially with digestive symptoms, such as abdominal pain, vomiting, and diarrhea. Only 2 patients with severe illness developed ARDS and required pediatric intensive care unit (PICU) admission, invasive mechanical ventilation therapy, and blood purification. All of them had gradually recovered and one patient had been discharged.

In our study, most patients had mild diseases including upper respiratory tract infection and mild pneumonia, which were less severe than symptoms reported in adult patients $\mathrm{s}^{[9,10]}$. Wang et a ${ }^{[10]}$ demonstrated that $26 \%$ of hospitalized adult patients with confirmed COVID-19 received ICU care, and mortality was $4.3 \%$. However, we only found 2 cases of children who received PICU care, and none of the patients in this study died from the disease. The occurrence of mild illness in pediatric patients has no clear explanation. Possible explanations may be related to the published data from other outbreaks of coronavirus infection, such as SARS and Middle East respiratory syndrome (MERS). It has been reported that children had a much milder and shorter course of SARS infection than adults, and only $3.3 \%$ of confirmed patients with MERS-CoV infection were aged less than 10 years $^{[14-17]}$. Therefore, children with COVID-19 had fewer infections and much milder illness than adults, which may be a feature of SARSCoV-2 infection. However, these findings need to be confirmed by large, well-designed studies.
However, there were 2 critical cases aged about 1 year in our study. It is known that secondary bacterial pneumonia caused the majority of the deaths in pandemic influenza ${ }^{[18]}$. In our study, one case had relatively high level of CRP with negative blood culture, and another one had the normal level of CRP with positive sputum culture. It has been established that several factors contributed to the low prevalence of positive blood or sputum culture results in pediatric pneumonia ${ }^{[19]}$. Furthermore, radiographic images of the 2 critical cases revealed bilateral lung consolidations. Thus, both of these 2 cases could be partly attributed to secondary bacterial infection.

In addition, patients who were hospitalized with COVID-19 had an uneven age distribution. The highest incidence of infection occurred in children was noted aged $<3$ years. This may be related to special respiratory tract structure infection, or immature immune system at this age, as well as a low compliance of wearing face masks in this age group. Chen et al ${ }^{[9]}$ reported that COVID-19 clustered within groups of humans in close contact. Children aged $<3$ years need constant care, which would increase the contact and the risk of exposure to SARS-CoV-2. In fact, a recent study reported nine infant cases and all infections occurred after the family members' infection with SARS$\mathrm{CoV}-2^{[12]}$.

Our study has several limitations. First, majority of our cases were still hospitalized for medical care, and we cannot therefore describe the full spectrum of this illness. Second, our study was limited by unified laboratory examinations. In some mild cases, serum electrolyte, cytokines, coagulation and renal function parameters were not evaluated. Third, we have included data for most but not all patients with laboratory-confirmed COVID-19 pediatric patients in Hubei, which resulted in a limited case size.

In conclusion, the present data reveal that children were susceptible to COVID-19 like adults, while the clinical presentations and outcomes were more favorable in children. However, children less than 3 years old accounted for majority cases and critical cases lied in this age group, which demanded extra attentions 
during home caring and hospitalization treatment.

\section{Conflict of Interest Statement}

All authors declare no conflict of interest.

\section{REFERENCES}

1 Bogoch II, Watts A, Thomas-Bachli A, et al. Pneumonia of Unknown Etiology in Wuhan, China: Potential for International Spread Via Commercial Air Travel. J Travel Med, 2020.doi: 10.1093/jtm/taaa008.

2 Lu H, Stratton CW, Tang YW. Outbreak of Pneumonia of Unknown Etiology in Wuhan China: the Mystery and the Miracle. J Med Virol, 2020.doi: 10.1002/jmv.25678.

3 Paules CI, Marston HD, Fauci AS. Coronavirus Infections-More Than Just the Common Cold. JAMA, 2020.doi: 10.1001/jama.2020.0757.

4 Hui DS, I AE, Madani TA, et al. The continuing 2019$\mathrm{nCoV}$ epidemic threat of novel coronaviruses to global health -- The latest 2019 novel coronavirus outbreak in Wuhan, China. Int J Infect Dis, 2020;91:264-266.doi: 10.1016/j.ijid.2020.01.009.

5 Chan JF, Kok KH, Zhu Z, et al. Genomic characterization of the 2019 novel human-pathogenic coronavirus isolated from a patient with atypical pneumonia after visiting Wuhan. Emerg Microbes Infect, 2020;9(1):221236.doi: 10.1080/22221751.2020.1719902.

6 National Health Commission of the People's Republic China, Novel coronavirus (2019-nCoV): situation. Accessed February 15, 2020. [. http://2019ncov.chinacdc. $\mathrm{cn} / 2019-\mathrm{nCoV} /$.

7 Huang C, Wang Y, Li X, et al. Clinical features of patients infected with 2019 novel coronavirus in Wuhan, China. Lancet. 2020.doi: 10.1016/S0140-6736(20)30183-5.

8 Zhu N, Zhang D, Wang W, et al. A Novel Coronavirus from Patients with Pneumonia in China, 2019. New Engl J Med, 2020.doi: 10.1056/NEJMoa2001017.

9 Chen N, Zhou M, Dong X, et al. Epidemiological and clinical characteristics of 99 cases of 2019 novel coronavirus pneumonia in Wuhan, China: a descriptive study. Lancet, 2020.doi: 10.1016/S01406736(20)30211-7.
10 Wang D, Hu B, Hu C, et al. Clinical Characteristics of 138 Hospitalized Patients With 2019 Novel Coronavirus-Infected Pneumonia in Wuhan, China. JAMA, 2020.doi: 10.1001/jama.2020.1585.

11 Chen F, Liu ZS, Zhang FR, et al. Frist case of severe childhood novel coronavirus pneumonia in China. Zhonghua Er Ke Za Zhi (Chinese), 2020;58(0):E005. doi: 10.3760/cma.j.issn.0578-1310.2020.0005.

12 Wei M, Yuan J, Liu Y, et al. Novel Coronavirus Infection in Hospitalized Infants Under 1 Year of Age in China. JAMA, 2020.doi: 10.1001/jama.2020.2131.

13 Pediatric Branch Of Hubei Medical Association PBOW. Recommendation for the diagnosis and treatment of novel coronavirus infection in children in Hubei (Trial version 1). Chin J Contemp Pediatr, 2020;2(22):96-99. doi: 10.7499/j.issn.1008-8830.2020.02.003.

14 Koh D, Sng J. Lessons from the past: perspectives on severe acute respiratory syndrome. Asia Pac J Public Health, 2010,22(3 Suppl):132S-136S.

15 Leung $\mathrm{CW}$, Chiu WK. Clinical picture, diagnosis, treatment and outcome of severe acute respiratory syndrome (SARS) in children. Paediatr Respir Rev, 2004,5(4):275-288.

16 Cheng VC, Lau SK, Woo PC, et al. Severe acute respiratory syndrome coronavirus as an agent of emerging and reemerging infection. Clin Microbiol Rev, 2007,20(4):660-694.

17 Alhamlan FS, Majumder MS, Brownstein JS, et al. Case characteristics among Middle East respiratory syndrome coronavirus outbreak and non-outbreak cases in Saudi Arabia from 2012 to 2015. Bmj Open, 2017,7(1):e011865.

18 Morens DM, Taubenberger JK, Fauci AS. Predominant role of bacterial pneumonia as a cause of death in pandemic influenza: implications for pandemic influenza preparedness. J Infect Dis, 2008,198(7):962970.

19 Fritz CQ, Edwards KM, Self WH, et al. Prevalence, Risk Factors, and Outcomes of Bacteremic Pneumonia in Children. Pediatrics, 2019,144(1):e20183090.

(Received Feb. 25, 2020; revised Mar. 10, 2020) 